\title{
Evolution of the dynamics of neutral superconductors between BCS and BEC regimes: the variational approach
}

\author{
Andrey V. Chubukov \\ Department of Physics, University of Minnesota, Minneapolis MN 55455, USA \\ E-mail: achubuko@umn.edu \\ Dmitry Mozyrsky \\ Theoretical Division (T-4), Los Alamos National Laboratory, Los Alamos NM 87545, USA \\ E-mail: mozyrsky@lanl.gov
}

Received January 24, 2018, published online April 25, 2018

\begin{abstract}
We use variational approach to analyze the evolution of the dynamics of a neutral s-wave superconductor between BCS and BEC regimes. We consider 2D case, when BCS-BEC crossover occurs already at weak coupling and is governed by the ratio of the two scales - the Fermi energy $E_{F}$ and the bound state energy for two fermions in a vacuum, $E_{0}$. BCS and BEC regimes correspond to $E_{F}>>E_{0}$ and $E_{F}<<E_{0}$, respectively. We compute the spectrum of low-energy bosonic excitations and show that the velocity of phase fluctuations remains $v_{F} / \sqrt{2}$ through BCS-BEC crossover. We also discuss the topological Ad term in the effective action.
\end{abstract}

PACS: 74.20.Fg Nanotubes;

74.25.Bt Thermodynamic properties;

74.78.-w Superconducting films and low-dimensional structures.

Keywords: s-wave, superconductor, Berry phase.

\section{Preface}

It is our great pleasure to present this article for a special issue in memory of Alexei Alexeevich Abrikosov. Alexei Alexeevich was one of the pioneers of field-theoretical approach to superconductivity and the co-author of the book [1] which educated at least two generations of physicists. He is also the father of vortex physics in superconductors. Our study is based on his works, and we hope it will be of interest to the readers of the special memorial issue of the Low Temperatures Physics.

\section{Introduction}

In this paper, we discuss the evolution of the dynamic properties of a neutral superconductor between BCS regime, when bound pairs of fermions condense immediately once they form, and Bose-Einstein condensation (BEC) regime, when bound pairs of fermions form at a higher $T_{\text {ins }}$ and condense at a smaller $T_{C}$. Experimental evidence for preformed pairs has been reported for high- $T_{C}$ cuprates [2] and, more recently, for Fe-based superconductor $\mathrm{FeSe}_{1-x} \mathrm{Te}_{x}$ (Ref. 3).
We consider a 2D s-wave superconductor and assume a rotational symmetry and $k^{2} /(2 m)$ fermionic dispersion. In 2D BCS-BEC crossover can be analyzed already within weak coupling, when calculations are under control [4-6]. Indeed, in 2D systems, two fermions form a bound state already at arbitrary small attraction $g$, with energy $2 E_{0}=2 \Lambda \mathrm{e}^{-2 /\left(N_{0} g\right)}$, where $N_{0}=m /(2 \pi)$ is the free particle density of states per spin in $2 \mathrm{D}$ and $\Lambda$ is the upper cutoff for the attraction [4-6]. In 3D systems, a bound state of two fermions emerges only once the interaction exceeds a certain cutoff, generally of the order of fermionic bandwidth [8].

The evolution of the static properties of a superconductor between BCS and BEC regimes has been extensively discussed in the condensed matter context $[4,5,7-23,42]$ and also for optical lattices of ultracold atoms [24,25]. The BCS-BEC crossover is determined by the interplay between $E_{0}$ and $E_{F}$ : the system is deep in the BCS regime when $E_{F} \gg E_{0}$ and deep in the BEC regime when $E_{0} \gg E_{F}$. In the BCS regime, the pairing instability temperature and superconducting $T_{C}$ are $T_{\text {ins }} \approx T_{C} \sim\left(E_{F} E_{0}\right)^{1 / 2}$, and the pairing gap $\Delta$ also scales as $\left(E_{F} E_{0}\right)^{1 / 2}$. In the BEC regime 
$T_{\text {ins }} \sim E_{0} / \log \left(E_{0} / E_{F}\right), T_{C} \sim E_{F}$, and $\Delta$ still scales as $\left(E_{F} E_{0}\right)^{1 / 2}$. The chemical potential $\mu(T=0)=E_{F}-E_{0}$, and it changes sign from positive to negative when $E_{0}$ becomes larger than $E_{F}$.

Here we focus on the evolution of the dynamical properties. We use variational approach, in which we assume that the order parameter $\Delta(\mathbf{r}, \tau)$ slowly fluctuates around its equilibrium value $\Delta_{0}$. We obtain the quadratic form in the variations $\Delta(\mathbf{r}, \tau)-\Delta_{0}$ and extract from it the spectrum of collective modes, including gapless Anderson-Bogolubov-Goldstone (AGB) phase mode. In the BCS regime, the velocity of AGB excitations, $v_{A G B}$, is the same as the velocity of a sound wave in the normal state: $v_{A G B}=v_{F} / \sqrt{2}$, where $v_{F}$ is Fermi velocity. We argue that the value $v_{A G B}$ remains $v_{F} / \sqrt{2}$ also in the BEC limit. Within this approach, we also identify the linear in frequency term, which corresponds to the $i \int d \mathbf{r} d \tau A \dot{\phi}(\mathbf{r}, \tau)$ term in the effective action. Such term is often referred to in the literature as the Berry phase term [26-31]. We obtain the variation of the prefactor $A$ with respect to the variation of $\Delta$. This allows us to express $A$ as $A=n / 2+C$, where $n$ is the density (which may depend on time), up to a constant $C$. A constant $C$ is irrelevant if the phase $\phi$ is defined globally, i.e., has a certain value at any point in space, because then a prefactor can be pulled out from the $\int d \mathbf{r} d \tau$, and the remaining term $\int d \mathbf{r} d \tau \dot{\phi}(\mathbf{r}, \tau)$ reduces to an irrelevant boundary term, which does not affect the equation of motion. However, if the phase is not defined globally, as in the case of a moving Abrikosov vortex with coordinates $X(\tau)$ and $Y(\tau), \int d \mathbf{r} d \tau \dot{\phi}(\mathbf{r}, \tau) \propto(\dot{X} Y-\dot{Y} X)$ does not reduce to a boundary term and does affect the equation of motion. In particular, it gives rise to an effective Magnus force acting on a vortex [26-37]. To obtain A exactly, one needs an alternative approach, in which one expands the effective action in terms of time derivatives of the order parameter [38]. This approach yields $C=0$ in the absence of impurity scattering.

The paper is organized as follows: in the next section we obtain, as a warm-up exercise, the expression for the condensation energy in the crossover between BCS and BEC regimes. In Sec. 4 we introduce the effective action of a superconductor in terms of its fluctuating order parameter $\Delta(\mathbf{r}, \tau)$. In Sec. 5 we derive the dispersion relation for the AGB mode by expanding in small variations of $\Delta$ relative to its equilibrium value. In Sec. 6 we obtain the term linear in the time derivative of the phase of the order parameter (the Berry phase term). We summarize our results in Sec. 7.

\section{The condensation energy}

We first consider the case when the order parameter $\Delta(\mathbf{r}, \tau)$ is a constant $\Delta_{0}$. In this situation the pairing Hamiltonian has a conventional form

$$
\begin{aligned}
& H_{0}=\sum_{k} \varepsilon_{k}\left(c_{k, \uparrow}^{\dagger} c_{k, \uparrow}+c_{k, \downarrow}^{\dagger} c_{k, \downarrow}\right)+ \\
& +\sum_{k}\left(\Delta_{0} c_{k, \uparrow}^{\dagger} c_{-k, \downarrow}^{\dagger}+\Delta_{0}^{*} c_{-k, \downarrow}^{\dagger} c_{k, \uparrow}\right),
\end{aligned}
$$

where operators $c_{\mathbf{k}, \sigma}^{\dagger}$ are Fourier transforms of $\psi_{\sigma}^{\dagger}(\mathbf{r})$, etc. and $\xi_{\mathbf{k}}=\xi_{-\mathbf{k}}=\varepsilon_{\mathbf{k}}-\mu, \varepsilon_{\mathbf{k}}=\mathbf{k}^{2} / 2 m$. The difference between the ground state energy $\left\langle H_{0}\right\rangle$ in a superconductor (with the chemical potential $\mu=E_{F}-E_{0}$ ) and in a normal state (with the chemical potential $\mu_{0}=E_{F}$ ) is the condensation energy $E_{\text {cond }}$.

The ground state energy is often separated into kinetic and potential energy parts, $E_{S C}=E_{\text {kin }}+E_{\text {pot }}$, although this splitting is a bit elusive for a quantum system of interacting fermions as, e.g., fermionic self-energy, which is a potential energy of a fermion in a field created by other fermions, contributes to the fermionic density $n(k)=\sum_{\alpha}\left\langle c_{k, \alpha}^{\dagger} c_{k, \alpha}\right\rangle$ and, hence, to the kinetic energy. We follow earlier works and define the kinetic energy as $E_{\text {kin }}=\sum_{k} \varepsilon_{k} n(k)$. The kinetic part of the ground state energy can be expressed via the normal Green's function as

$$
E_{\mathrm{kin}}=-2 \sum \sum_{\mathbf{k}, \omega}\left[\frac{i \omega+\xi_{\mathbf{k}}}{\omega^{2}+\Delta_{0}^{2}+\xi_{\mathbf{k}}^{2}}\right],
$$

where at $T=0$ and in thermodynamic limit

$$
\sum_{\mathbf{k}, \omega}=S \int d^{2} k d \omega /(2 \pi)^{3}=S N_{0} \int d \varepsilon d \omega /(2 \pi),
$$

where $S$ is the area of a 2D sample.

The potential energy is

$$
E_{\mathrm{pot}}=-\Delta_{0} \sum_{\mathbf{k}, \omega} \frac{\Delta_{0}}{\omega^{2}+\Delta_{0}^{2}+\xi_{\mathbf{k}}^{2}}
$$

For a system with weak attraction $g$, the self-consistent equation on $\Delta_{0}$ is

$$
\frac{\Delta_{0}}{g}=\frac{1}{S} \sum_{\omega, \mathbf{k}} \frac{\Delta_{0}}{\omega^{2}+\Delta_{0}^{2}+\xi_{\mathbf{k}}^{2}} .
$$

Carrying out integration over the Matsubara frequency and over $\xi_{k}$ up to the cutoff $\Lambda$, and using $E_{0}=\Lambda \exp \left(-2 / N_{0} g\right)$, we obtain the algebraic equation [4-6]

$$
\sqrt{\mu^{2}+\Delta_{0}^{2}}-\mu=2 E_{0},
$$

which defines $\Delta_{0}$ in terms of the actual chemical potential $\mu$ and the two-particle bound state energy $E_{0}$. The selfconsistency equation for $\mu$ follows from the condition that the total number of fermions, including bound pairs, is conserved [5]. This yields 


$$
\sqrt{\mu^{2}+\Delta_{0}^{2}}+\mu=2 E_{F}
$$

The solution of Eqs. (5), (6) is

$$
\mu=E_{F}-E_{0}, \Delta_{0}=2 \sqrt{E_{F} E_{0}} .
$$

Like we said, BCS-BEC crossover occurs when $E_{0}$ becomes comparable to the Fermi energy, $E_{F}$. BCS behavior is realized when the bound state energy $E_{0}$ is much smaller than $E_{F}$, and BEC behavior is realized when $E_{0} \gg E_{F}$. A negative $\mu$ at $E_{F}<E_{0}$ implies that the Fermi momentum $k_{F}$, defined as position of the minimum of the fermionic dispersion $E_{k}=\sqrt{\left(\varepsilon_{k}-\mu\right)^{2}+\Delta_{0}^{2}}$, is zero [12]. When the particle density is small, $E_{F}$ is small compared to $\Lambda$, and the crossover occurs at small $E_{0} / \Lambda$, i.e., already at weak coupling, when $N_{0} g \ll 1$.

To obtain the condensation energy in BCS-BEC crossover regime, we write

$$
\begin{gathered}
E_{S C}=-N_{0} S \int \frac{d \varepsilon d \omega}{(2 \pi)} \frac{2 \varepsilon(i \omega+(\varepsilon-\mu))+\Delta_{0}^{2}}{\omega^{2}+\Delta_{0}^{2}+(\varepsilon-\mu)^{2}}, \\
E_{\text {norm }}=N_{0} S \int \frac{d \varepsilon d \omega}{(2 \pi)} \frac{2 \varepsilon}{i \omega-\left(\varepsilon-\mu_{0}\right)} .
\end{gathered}
$$

Evaluating the integrals separately for $E_{F}>E_{0}$, when $\mu>0$, and for $E_{F}<E_{0}$, when $\mu<0$, and in both cases using Eq. (7) to relate $\mu$ with $\Delta_{0}$, we obtain after a straightforward algebra that the condensation energy

$$
E_{\text {cond }}=-S N_{0} \Delta_{0}^{2} f\left(\frac{\mu}{\Delta}, \frac{\mu_{0}}{\Delta}\right),
$$

where

$$
f(x, y)=\frac{1}{4}+y^{2}-\frac{x\left(\sqrt{x^{2}+1}+x\right)}{2} .
$$

Using $\sqrt{x^{2}+1}+x=2 y$ (Eq. (6)) we obtain

$$
f(x, y)=\frac{1}{4}+y(y-x) \text {. }
$$

Using next $y(x-y)=\mu_{0}\left(\mu_{0}-\mu\right) / \Delta^{2}=E_{F} E_{0} / \Delta^{2}=1 / 4$, we find

$$
f(x, y)=\frac{1}{4}+\frac{1}{4}=\frac{1}{2}
$$

Substituting into (9), we obtain that

$$
E_{\text {cond }}=-S N_{0}\left(2 E_{F} E_{0}\right)=-S N_{0} \frac{\Delta_{0}^{2}}{2}
$$

in the whole BCS-BEC crossover region.

A remark is in order about the order of integration over frequency and dispersion. The order does not matter as long as the integral over $\varepsilon_{k}$ is taken within finite limits, i.e., one can do $\int d \omega d \varepsilon$ in any order and the result will be the same. The situation gets more tricky when the integration over $\varepsilon$ is extended to infinite limit. The most extreme case here is BCS limit, where $\mu \gg \Delta$, and the integration over $\xi=\varepsilon-\mu$ can be formally extended to $\int_{-\infty}^{\infty} d \xi$. In the BCS limit, the difference between $\mu$ and $\mu_{0}$ is irrelevant, to leading order in $\Delta / \mu$, and $E_{\text {cond }}$ can be expressed as

$$
E_{\text {cond }}=-S N_{0} \Delta_{0}^{2} \int \frac{d \omega d \xi}{2 \pi} \frac{\omega^{2}-\xi^{2}}{\left(\omega^{2}+\xi^{2}\right)\left(\omega^{2}+\xi^{2}+\Delta_{0}^{2}\right)} \text {. }
$$

At small $\omega$ and $\xi$, the would be divergence of the integrand is regularized by $\Delta_{0}$, but at large $\omega$ and $\xi$, the integrand scales as $\left(\omega^{2}-\xi^{2}\right) /\left(\omega^{2}+\xi^{2}\right)^{2}$, i.e., the 2D integral is marginal by power counting. Such an integral is nonsingular, but its value depends on how the integration is performed. If we treat $\omega$ and $\xi$ as “equivalent" variables and evaluate the integral using polar coordinates, the integral vanishes because of $\omega^{2}-\xi^{2}$ in the numerator. If we integrate over $\omega$ first or over $\xi$ first, both times in infinite limits, the result is finite, but obviously of a different sign. Because at $T=0$ the integration over Matsubara frequency $\omega$ truly goes from $-\infty$ to $+\infty$, while the integration over $\xi$ actually holds in finite limits, which can only approximately be extended to $\pm \infty$, the correct way to evaluate the integral in (14) is to integrate over frequency first. Integrating, we obtain

$$
E_{\text {cond }}=-\frac{1}{2} S N_{0} \Delta_{0}^{2} \int \frac{d \xi}{\sqrt{\xi^{2}+\Delta_{0}^{2}}} \frac{\Delta_{0}^{2}}{\left(|\xi|+\sqrt{\xi^{2}+\Delta^{2}}\right)^{2}} .
$$

The integrand scales as $1 /|\xi|^{3}$ at large $|\xi|$, i.e., the integral converges and can be evaluated in infinite limits. By rescaling $\xi=\Delta_{0} x$, one can immediately verify that the integral does not depend on $\Delta_{0}$, and (15) reduces to

$$
E_{\text {cond }}=-S N_{0} \Delta_{0}^{2} \int_{0}^{\infty} \frac{d x}{\sqrt{x^{2}+1}}\left(\sqrt{x^{2}+1}-x\right)^{2}=-\frac{1}{2} S N_{0} \Delta_{0}^{2} \text {. }
$$

This agrees with (13).

One can further check that the variation of the energy is exactly the same as the variation of the chemical potential $n \delta \mu=n\left(\mu-E_{F}\right)=2 N_{0} E_{F}\left(E_{0}+E_{F}-E_{F}\right) \equiv N_{0} \Delta_{0}^{2} / 2$. As a result, $E-\mu N$ (equal to the Grand potential at $T=0$ ) does not change between the normal and the superconducting state.

Note in passing that the self-consistency analysis can be straightforwardly extended to a finite $T$. In the BCS regime, the onset temperatures $T_{p}$ for the pairing and $T_{C}$ for superconductivity (i.e., for pair coherence) are comparable and both scale as $\sqrt{E_{F} E_{0}}$ (more precisely, $T_{p}$ almost coincides with the mean-field $T_{c}$, while the actual $T_{c}$ of Berezinskii-Kosterlitz-Thouless transition is smaller by a numerical factor). In the BEC regime the two temperatures differ strongly: $T_{p} \sim E_{0} / \log \left(E_{0} / E_{F}\right) \gg E_{F}$, while $T_{C} \sim E_{F}$, i.e., the ratio $T_{C} / T_{p}$ vanishes at $E_{F} \rightarrow 0$ (see, e.g., Ref. 6). When $E_{F} \gg E_{0}, \Delta_{0}=1.76 T_{\text {ins }}$, like in BCS theory, when $E_{F} \ll E_{0}, \Delta_{0}$ is much larger than $T_{C}$, but much smaller than $T_{p}$. 


\section{The effective action}

The effective action for an order parameter of an s-wave superconductor can be obtained by departing from a microscopic model with local four-fermion attractive interaction $-g(g>0)$ and decoupling four-fermion interaction via Hubbard-Stratonovich transformation [39], by introducing the pairing field $\Delta(r, \tau)$. This procedure is well documented, and we just quote the results.

The partition function $Z$ is expressed via the integral over the Grassmann fields as

$$
Z=\int d \psi d \bar{\psi} \mathrm{e}^{-S[\psi, \bar{\psi}]},
$$

where $\psi=\psi_{\alpha}(\mathbf{r}, \tau)$ and $\bar{\psi}=\bar{\psi}_{\alpha}(\mathbf{r}, \tau)$ are spin-full coordinate and time dependent Grassmann fields, and

$$
S[\psi, \bar{\psi}]=\int d \mathbf{r} d \tau\left(\bar{\psi}_{\alpha}(\mathbf{r}, \tau) \partial_{\tau} \psi_{\alpha}(\mathbf{r}, \tau)+H[\psi, \bar{\psi}]\right) .
$$

Here $\tau$ is the imaginary (Matsubara) time $\tau=i t$ and

$$
\begin{gathered}
H[\psi, \bar{\psi}]=\left[\psi_{\sigma}^{\dagger}(\mathbf{r}, \tau)\left(-\frac{\nabla^{2}}{2 m}-\mu\right) \psi_{\sigma}(\mathbf{r}, \tau)\right]- \\
-g \psi_{\uparrow}^{\dagger}(\mathbf{r}, \tau) \psi_{\downarrow}^{\dagger}(\mathbf{r}, \tau) \psi_{\downarrow}(\mathbf{r}, \tau) \psi_{\uparrow}(\mathbf{r}, \tau) .
\end{gathered}
$$

The four-fermion interaction is decoupled by Hubbard-Stratonovich transformation

$$
\mathrm{e}^{\frac{a x^{2}}{2}}=\frac{1}{\sqrt{2 \pi a}} \int d y \mathrm{e}^{\left(-\frac{y^{2}}{2 a}+y x\right)} .
$$

In our case we introduce two Hubbard-Stratonovich fields $\Delta(\mathbf{r}, \tau)$ and $\Delta^{*}(\mathbf{r}, \tau)$ and rewrite the partition function as

$$
Z=\int d \psi d \bar{\psi} d \Delta d \Delta^{*} \mathrm{e}^{-S\left[\psi, \bar{\psi}, \Delta, \Delta^{*}\right]},
$$

where now

$$
\begin{gathered}
S\left[\psi, \bar{\psi}, \Delta, \Delta^{*}\right]= \\
=\int d \mathbf{r} d \tau\left(\bar{\psi}_{\alpha}(\mathbf{r}, \tau) \partial_{\tau} \psi_{\alpha}(\mathbf{r}, \tau)+\frac{|\Delta(\mathbf{r}, \tau)|^{2}}{g}+H\left[\psi, \bar{\psi}, \Delta, \Delta^{*}\right]\right),
\end{gathered}
$$

and

$$
\begin{aligned}
& H\left[\psi, \bar{\psi}, \Delta, \Delta^{*}\right]=\left[\bar{\psi}_{\sigma}(\mathbf{r}, \tau)\left(-\frac{\nabla^{2}}{2 m}-\mu\right) \psi_{\sigma}(\mathbf{r}, \tau)+\right. \\
+ & \left.\Delta(\mathbf{r}, \tau) \bar{\psi}_{\uparrow}(\mathbf{r}, \tau) \bar{\psi}_{\downarrow}(\mathbf{r}, \tau)+\Delta^{*}(\mathbf{r}, \tau) \psi_{\downarrow}(\mathbf{r}, \tau) \psi_{\uparrow}(\mathbf{r}, \tau)\right] .
\end{aligned}
$$

The action $S\left[\psi, \bar{\psi}, \Delta, \Delta^{*}\right]$ can be re-expressed in a more compact form by introducing Gorkov-Nambu spinor $\psi=\left[\psi_{\uparrow}, \psi_{\downarrow}^{\dagger}\right]^{T}$. Then

$$
\begin{gathered}
S\left[\psi, \bar{\psi}, \Delta, \Delta^{*}\right]=\int d \mathbf{r} d \tau \frac{|\Delta(\mathbf{r}, \tau)|^{2}}{g}- \\
-\int d \mathbf{r} d \tau \int d \mathbf{r}^{\prime} d \tau^{\prime} \bar{\psi}(\mathbf{r}, \tau) G^{-1}\left(\mathbf{r}, \tau ; \mathbf{r}^{\prime}, \tau^{\prime}\right) \psi\left(\mathbf{r}^{\prime}, \tau^{\prime}\right),
\end{gathered}
$$

where the inverse Green's function $G^{-1}\left(\mathbf{r}, \tau ; \mathbf{r}^{\prime}, \tau^{\prime}\right)$ is given by

$$
G^{-1}\left(\mathbf{r}, \tau ; \mathbf{r}^{\prime}, \tau^{\prime}\right)=\left[-\partial_{\tau}-\hat{K}(\mathbf{r})-\hat{\Delta}(\mathbf{r}, \tau, \lambda)\right] \delta\left(\mathbf{r}-\mathbf{r}^{\prime}\right) \delta\left(\tau-\tau^{\prime}\right),
$$

with

$$
\hat{K}(\mathbf{r})=\left[\begin{array}{cc}
-(1 / 2 m) \nabla^{2}-\mu & 0 \\
0 & (1 / 2 m) \nabla^{2}+\mu
\end{array}\right],
$$

and

$$
\hat{\Delta}(\mathbf{r}, \tau)=\left[\begin{array}{cc}
0 & \Delta(\mathbf{r}, \tau) \\
\Delta^{*}(\mathbf{r}, \tau) & 0
\end{array}\right] .
$$

Integrating over $\psi$ and $\bar{\psi}$ we then obtain

$$
Z=\int d \Delta d \Delta^{*} \mathrm{e}^{-S\left[\Delta, \Delta^{*}\right]}
$$

and

$$
\begin{gathered}
S\left[\Delta, \Delta^{*}\right]=\int d \mathbf{r} d \tau \frac{|\Delta(\mathbf{r}, \tau)|^{2}}{g}- \\
-\int d \mathbf{r} d \tau \int d \mathbf{r}^{\prime} d \tau^{\prime} \operatorname{Tr} \log \hat{G}^{-1}\left(\mathbf{r}, \tau ; \mathbf{r}^{\prime}, \tau^{\prime}\right) .
\end{gathered}
$$

In Fourier space (momentum $k$ and Matsubara frequency $\omega$ )

$$
\hat{G}^{-1}(k, \omega)=\left[\begin{array}{cc}
i \omega-\xi_{k} & \Delta^{*}(k, \omega) \\
\Delta^{*}(k, \omega) & i \omega+\xi_{k}
\end{array}\right] .
$$

\section{The dynamics of phase fluctuations}

We now expand the action in small variations of the order parameter around a constant value, $\delta \Delta(\mathbf{r}, \tau)=\Delta(\mathbf{r}, \tau)-$ $-\Delta_{0} \delta(\mathbf{r}) \delta(\tau)$.. The action to the second order in $\delta \Delta$ has been obtained in the BCS-BEC crossover regime in Ref. 40 , and our expression for the quadratic form in $\delta \Delta$ in the action agrees with theirs. The authors of Ref. 40, however, didn't extract the spectrum of the ABG mode from their data, nor they extracted the linear in $\Omega$ (i.e., $\dot{\phi}$ ) term in the action. Collecting all second-order contributions and Fourier transforming to momentum and Matsubara frequency space, we obtain the variation of $\mathcal{S}$ in the form

$$
\delta \mathcal{S}=\frac{1}{2} \sum_{\mathbf{q}, \Omega}\left[A_{+}\left|\delta \Delta_{+}\right|^{2}+A_{-}\left|\delta \Delta_{-}\right|^{2}+B\left(\delta \Delta_{+} \delta \Delta_{-}+\text {h.c. }\right)\right],
$$

where

$$
\begin{aligned}
& \delta \Delta_{+}=\delta \Delta(\mathbf{q}, \Omega)=\int d \mathbf{r} d \tau \delta \Delta(\mathbf{r}, t) \mathrm{e}^{-i \mathbf{q} \mathbf{r}+i \Omega \tau}, \\
& \delta \Delta_{-}=\delta \Delta(-\mathbf{q},-\Omega)=\int d \mathbf{r} d \tau \delta \Delta(\mathbf{r}, t) \mathrm{e}^{i \mathbf{q} \mathbf{r}-i \Omega \tau}
\end{aligned}
$$

and 


$$
\begin{gathered}
A_{ \pm}=\frac{1}{g}-\sum_{k, \omega} \varepsilon_{\mathbf{k}} \frac{\left(i(\omega \pm \Omega / 2)+\xi_{\mathbf{k} \pm \mathbf{q} / 2}\right)\left(-i(\omega \mp \Omega / 2)+\xi_{-\mathbf{k} \pm \mathbf{q} / 2}\right)}{\left((\omega \pm \Omega / 2)^{2}+\Delta_{0}^{2}+\xi_{\mathbf{k} \pm \mathbf{q} / 2}^{2}\right)\left((\omega \mp \Omega / 2)^{2}+\Delta_{0}^{2}+\xi_{-\mathbf{k} \pm \mathbf{q} / 2}^{2}\right)} \\
B=\sum_{\mathbf{k}, \omega} \frac{\Delta_{0}^{2}}{\left((\omega+\Omega / 2)^{2}+\Delta_{0}^{2}+\xi_{\mathbf{k}+\mathbf{q} / 2}^{2}\right)\left((\omega-\Omega / 2)^{2}+\Delta_{0}^{2}+\xi_{-\mathbf{k}+\mathbf{q} / 2}^{2}\right)}
\end{gathered}
$$

where, we remind, $\xi_{k}=\varepsilon_{k}-\mu=k^{2} /(2 m)-\mu$.

The excitation spectrum is obtained from the condition $A_{+} A_{-}=B^{2}$, or, equivalently

$$
\left(\frac{A_{+}+A_{-}}{2}+B\right)\left(\frac{A_{+}+A_{-}}{2}-B\right)-\left(\frac{A_{+}-A_{-}}{2}\right)^{2}=0 .
$$

Evaluating the frequency and momentum integrals, we obtain

$$
\begin{aligned}
& \frac{A_{+}+A_{-}}{2}-B=\frac{N_{0}}{8 \Delta_{0}^{2}}\left[\Omega^{2}\left(1+\frac{\mu}{\sqrt{\mu^{2}+\Delta_{0}^{2}}}\right)+\right. \\
& \left.+\frac{\mathbf{q}^{2}}{m}\left(\mu\left(1+\frac{\mu}{\sqrt{\mu^{2}+\Delta_{0}^{2}}}\right)+\frac{\Delta_{0}^{2}}{\sqrt{\mu^{2}+\Delta_{0}^{2}}}\right)\right]= \\
& =\frac{N_{0}}{4 \Delta_{0}^{2}}\left[\Omega^{2} \frac{E_{F}}{E_{0}+E_{F}}+\frac{\mathbf{q}^{2}}{m} E_{F}\right] \text {, } \\
& \frac{A_{+}+A-}{2}+B=\frac{N_{0}}{2}\left(1+\frac{\mu}{\sqrt{\mu^{2}+\Delta_{0}^{2}}}\right)+\ldots=\frac{N_{0} E_{F}}{E_{0}+E_{F}}+\ldots \\
& \frac{A_{+}-A}{2}=i \Omega A, A=-\frac{N_{0}}{4} \frac{1}{\sqrt{\mu^{2}+\Delta_{0}^{2}}}=-\frac{N_{0}}{4} \frac{1}{E_{F}+E_{0}} \text {, }
\end{aligned}
$$

where dots stand for $\Omega$ and $q$-dependent terms in $\left(A_{+}+A_{-}\right) / 2+B$, which we do not need. Substituting this into (31), we obtain from (31)

$$
\frac{N_{0}^{2}}{16 E_{0}\left(E_{F}+E_{0}\right)}\left[\Omega^{2}+\frac{v_{F}^{2}}{2} \mathbf{q}^{2}\right]=0 \text {. }
$$

Converting this last expression into real frequencies $(\Omega \rightarrow-i \Omega)$, we see that the excitation spectrum has a gapless mode, whose velocity remains $v_{F} / \sqrt{2}$, no matter what the ratio of $E_{0} / E_{F}$ is.

To see that this mode corresponds to phase fluctuations, we split the complex variations $\delta \Delta_{+}$and $\delta \Delta_{-}$into real and imaginary parts $\delta \Delta_{ \pm}^{\prime}$ and $\delta \Delta_{ \pm}^{\prime \prime}$ and re-express the action in Eq. (28) as

$$
\begin{aligned}
\delta \mathcal{S} & =\frac{1}{2} \sum_{q, \Omega} A_{+}\left(\delta \Delta_{+}^{\prime} \frac{B}{A_{+}} \delta \Delta_{-}^{\prime}\right)^{2}+\left(\delta \Delta_{-}^{\prime}\right)^{2}\left(A_{-}-\frac{B^{2}}{A_{+}}\right)+ \\
& +\frac{1}{2} \sum_{q, \Omega} A_{+}\left(\delta \Delta_{-}^{\prime \prime} \frac{B}{A_{+}} \delta \Delta_{-}^{\prime \prime}\right)^{2}+\left(\delta \Delta_{-}^{\prime \prime}\right)^{2}\left(A_{-}-\frac{B^{2}}{A_{+}}\right) .
\end{aligned}
$$

This expression shows that there are gapped and gapless modes. Suppose that the equilibrium value of $\Delta=\Delta_{0}$ is real. Then longitudinal variation $\delta \Delta(r, t)$ in coordinate is real and transverse one is imaginary. Using Eq. (29) we find that for longitudinal gap fluctuations $\delta \Delta_{+}^{\prime}=-\delta \Delta_{-}^{\prime}$ and $\delta \Delta_{+}^{\prime \prime}=\delta \Delta_{-}^{\prime \prime}$, while for transverse (phase) fluctuations $\delta \Delta_{+}^{\prime}=-\delta \Delta_{-}^{\prime}$ and $\delta \Delta_{+}^{\prime \prime}=\delta \Delta_{-}^{\prime \prime}$. Using now the fact that at $\Omega=0$ and $q=0, A_{+}=A_{-}=B$, we immediately find from (31) that longitudinal fluctuations are gapped and phase fluctuations are gapless, as they should be.

The $q^{2}$ term in the integrand in Eq. (28) for $\delta \mathcal{S}$ determines the phase stiffness. Taking $q^{2}$ term from $A_{+}+A_{-}$ in (29) and expressing $\Delta(r, \tau)$ as $\Delta(r, \tau)=\Delta_{0} \mathrm{e}^{i r \nabla \phi}$, such that $\delta \Delta(q, \Omega)=\Delta_{0} \delta(\mathbf{q}-\nabla \phi)$, we obtain

$$
\delta \mathcal{S}=\int d \mathbf{r} \frac{n}{8 m}(\nabla \phi)^{2}=\frac{N_{0} E_{F}}{4 m}(\nabla \phi)^{2} .
$$

We see that the phase stiffness also does not change in BCS-BEC crossover and remains the same as in BCS limit [41].

The expression for $\delta \mathcal{S}$, Eqs. (28) and (30), have been obtained in [40], but the excitation spectrum have not been obtained there, although the dispersion of phase fluctuations at arbitrary $E_{0} / E_{F}$ can be extracted from Eq. (26) in that paper.

\section{The term linear in $\partial_{\tau} \phi$ in the action}

The prefactors $A_{+}$and $A_{-}$contain the piece linear in $\Omega$, with opposite signs. The corresponding term in the action is

$$
\begin{gathered}
\delta \mathcal{S}_{\text {linear }}=-i \frac{N_{0}}{8 \sqrt{\mu^{2}+\Delta_{0}^{2}}} \times \\
\times \sum_{q, \Omega} \Omega\left[\left(\left(\delta \Delta_{+}^{\prime}\right)^{2}-\left(\delta \Delta_{-}^{\prime}\right)^{2}\right)+\left(\left(\delta \Delta_{+}^{\prime \prime}\right)^{2}-\left(\delta \Delta_{-}^{\prime \prime}\right)^{2}\right)\right]= \\
=-i \frac{N_{0}}{8 \sqrt{\mu^{2}+\Delta_{0}^{2}}} \times \\
\times \sum_{q, \Omega} \Omega\left[\left(\delta \Delta_{+}^{\prime}+\delta \Delta_{-}^{\prime}\right)\left(\delta \Delta_{+}^{\prime}-\delta \Delta_{-}^{\prime}\right)+\left(\delta \Delta_{+}^{\prime \prime}+\delta \Delta_{-}^{\prime \prime}\right)\left(\delta \Delta_{+}^{\prime \prime}-\delta \Delta_{-}^{\prime \prime}\right)\right] .
\end{gathered}
$$

For definiteness, let's assume that $\delta \Delta_{ \pm}$are real, i.e., $\delta \Delta_{ \pm}=\delta \Delta_{+}^{\prime}$. The transverse gap variation $\delta \Delta_{+}^{\prime}-\delta \Delta_{-}^{\prime}$ can be expressed as the time variation of the phase of superconducting gap $\Delta(r, \tau)=\Delta_{0} \mathrm{e}^{i \dot{\phi} \tau}=\Delta_{0}(1+i \dot{\phi} \tau+\ldots)$, where $\phi=\phi(\mathbf{r}, \tau)$ and $\dot{\phi}=\partial \phi / \partial \tau$. Taking the Fourier transform we find 


$$
\delta \Delta_{+}^{\prime}-\delta \Delta_{-}^{\prime}=-2 \dot{\phi} \Delta_{0} \int \tau^{\prime} \sin \Omega \tau^{\prime} d \tau^{\prime} .
$$

The longitudinal gap variation can be approximated by its $q=0$ and $\Omega=0$ value $\delta \Delta^{\prime}=\int d \mathbf{r} d \tau \delta \Delta^{\prime}(\mathbf{r}, \tau)$. Substituting this into Eq. (36) we obtain

$$
\delta \mathcal{S}_{\text {linear }}=i Q \int d \mathbf{r} d \tau \frac{N_{0} \Delta_{0}}{2 \sqrt{\mu^{2}+\Delta_{0}^{2}}} \dot{\phi} \delta \Delta^{\prime}(\mathbf{r}, \tau)
$$

where

$$
Q=\int \frac{d \Omega d \tau^{\prime}}{2 \pi} \Omega \tau^{\prime} \sin \Omega \tau^{\prime}
$$

The integral contains the universal contribution $Q=(2 / \pi) \times$ $\times \int d \Omega(\sin \Omega / \Omega)=1$ and parasitic high-energy contribution, which vanishes under proper regularization. Substituting $Q=1$ into (38) we obtain

$$
\delta \mathcal{S}_{\text {linear }}=i \int d \mathbf{r} d \tau \frac{N_{0} \Delta_{0}}{2 \sqrt{\mu^{2}+\Delta_{0}^{2}}} \dot{\phi} \delta \Delta^{\prime}(\mathbf{r}, \tau) .
$$

We now observe that to leading order in $\delta \Delta^{\prime}(\mathbf{r}, \tau)$, $\Delta_{0} \delta \Delta^{\prime}(\mathbf{r}, \tau) / \sqrt{\mu^{2}+\Delta_{0}^{2}}$ is the variation of $\sqrt{\mu^{2}+|\Delta(\mathbf{r}, \tau)|^{2}}$ over $\Delta$, keeping $\mu$ constant. As the consequence, Eq. (40) can be viewed as the variation of

$$
\mathcal{S}_{\text {linear }}=i \frac{N_{0}}{2} \int d \mathbf{r} d \tau\left[\sqrt{\mu^{2}+\Delta_{0}^{2}}+\bar{C}(\mu)\right] \frac{\partial \phi(\mathbf{r}, \tau)}{\partial \tau}=i A \int d \mathbf{r} d \tau \dot{\phi},
$$

where

$$
A=\frac{N_{0}}{2}\left[\sqrt{\mu^{2}+\Delta_{0}^{2}}+\bar{C}(\mu)\right]
$$

and $\bar{C}(\mu)$ is some unknown constant, which depends on $\mu$. Using $n=2 N_{0} E_{F}=N_{0}\left(\sqrt{\mu^{2}+\Delta_{0}^{2}}+\mu\right)$, we can rewrite (42) as

$$
A=\frac{n}{2}+C(\mu)
$$

where $C(\mu)$ is some other constant. This constant cannot be obtained using the variational approach. In Ref. 38 we obtained a systematic expansion of the action in terms of the derivatives of the order parameter over the imaginary time. In this approach we computed the $A$ term explicitly and found that $C=0$ in the limit when the energy difference between discrete levels in the vortex core well exceeds a scattering rate due to impurities.

\section{Conclusion}

In this paper we analyzed the evolution of the low-frequency dynamics of collective excitations of an s-wave neutral superconductor at zero temperature, between BCS and $\mathrm{BEC}$ regimes. The two regimes correspond to small and large ratio of $E_{0} / E_{F}$, respectively, where $E_{F}$ is the Fermi energy, and $E_{0}$ is the bound state energy for two particles. In 2D, bound state develops already at weak coupling, what allows one to analyze the crossover without including strong coupling renormalizations. We found that the phase velocity of the collective excitations remains $v_{F} / \sqrt{2}$ through the BCS-BEC crossover. The superconducting stiffness (the prefactor for $(\nabla \phi)^{2}$ term in the action) also does not change through the BCS-BEC crossover and remains equal to $N_{0} E_{F} /(4 m)=n /(8 m)$, as in BCS limit. The action also contains the term linear in time derivative of $\phi-$ the Berry phase term. When $\phi$ is defined globally, i.e., has a certain value at any point in space, such term reduces to a boundary term and does not contribute to equation of motion, if the density is homogeneous. If $n=n(\tau)$, the linear in $\dot{\phi}$ term in the action is $\mathcal{S}_{\text {linear }}=(i / 2) \int d \mathbf{r} \int d \tau n(\tau) \dot{\phi}$, where $\phi=\phi(\mathbf{r}, \tau)$. For the case of a moving vortex with coordinates $X(\tau)$ and $Y(\tau)$, $\phi$ is not uniquely defined at the center of the vortex core, i.e., for $x \rightarrow X$ and $y \rightarrow Y$. In this situation $\int d \mathbf{r} d \tau \dot{\phi} \propto \int d \tau(\dot{X} Y-Y \dot{X})$, which does not reduce to the boundary term. Then the Berry phase term in the action contributes to equation of motion even when the prefactor is a constant, and it becomes relevant to find the exact prefactor $A=n / 2+C$. The exact prefactor $A$ has been computed by expanding the action in time derivatives of the order parameter [38]. This calculation yields $C=0$.

Our results for the expansion of the effective action in terms of $\delta \Delta^{\prime}$ can be straightforwardly extended to other symmetries of the order parameter and to non-Galileaninvariant dispersion. One can also use our formulas to obtain terms with higher-order derivatives (these are terms with higher powers of $\Omega$ and $q$ in the integrand of Eq. (28)).

\section{Acknowledgements}

We thank I. Aleiner, J.J. Dziarmaga, I. Martin, M. Stone, J. Sauls, D. Solenov, and G.E. Volovik for valuable discussions. The work at Los Alamos was performed under the auspices of the United States Department of Energy under Contract DE5AC52-06NA25396 and supported by LDRD and BES Grant No's. 20170460ER and E3B7, respectively. The work by AVC was supported by the Office of Basic Energy Sciences U.S. Department of Energy under award DE-SC0014402. AVC is thankful to KITP at UCSB, where part of the work has been done. KITP is supported by NSF grant PHY-1125915.

1. A.A. Abrikosov, L.P. Gor'kov, and I.E. Dzyaloshinski, Methods of Quantum Field Theory on Statistical Physics, Dover Publications, New York (1975).

2. see e.g., J.C. Campuzano, M.R. Norman, and M. Randeria, in: Physics of Superconductors, Vol. II, K.H. Bennemann and J.B. Ketterson (eds.), Springer, Berlin (2004), p. 167; Lu Li, Yayu Wang, and N.P. Ong, Phys. Rev. B 87, 056502 (2013) and references therein; D.S. Dessau, private communication.

3. K. Okazaki, Sci. Rep. 4, 4109 (2014); S. Kasahara, PNAS 111, 16309 (2014); S. Rinott, Sci. Adv. 3, e1602372 (2017). 
4. K. Miyake, Prog. Theor. Phys. 69, 1794 (1983).

5. M. Randeria, J.-M. Duan, and L.-Y. Shieh, Phys. Rev. Lett. 62, 981 (1989); Phys. Rev. B 41, 327 (1990).

6. A.V. Chubukov, I. Eremin, and D.V. Efremov, Phys. Rev. B 93, 174516 (2016).

7. V.M. Loktev, R.M. Quick, and S.G. Sharapov, Phys. Rep. 349, 1 (2001).

8. C.A.R. Sa de Melo, M. Randeria, and J.R. Engelbrecht, Phys. Rev. Lett. 71, 3202 (1993); J.R. Engelbrecht, M. Randeria, and C.A.R. Sa de Melo, Phys. Rev. B 55, 15153 (1997).

9. P. Noziéres and S. Schmitt-Rink, J. Low Temp. Phys. 59, 195 (1985).

10. M. Drechsler and W. Zwerger, Ann. Phys. 1, 15 (1992).

11. Y. Ohashi and A. Griffin, Phys. Rev. A 67, 033603 (2003)

12. Y.L. Loh, M. Randeria, N. Trivedi, C.-C. Chang, and R. Scalettar, arXiv:1507.05641.

13. see M.Y. Kagan, Modern Trends in Superconductivity and Superfluidity, Lecture Notes in Physics, 874, Springer, Heidelberg/Germany (2013) and references therein.

14. V.B. Geshkenbein, L.B. Ioffe, and A.I. Larkin, Phys. Rev. B 55, 3173 (1997).

15. B. Tobijaszewska and R. Micnas, Acta Physica Polonica A 97, 393 (2000).

16. L. Benfatto, A. Toschi, S. Caprara, and C. Castellani, Phys. Rev. B 64, 140506(R) (2001); L. Benfatto, S. Caprara, C. Castellani, A. Paramekanti, and M. Randeria, Phys. Rev. B 63, 174513 (2001); L. Benfatto, A. Toschi, and S. Caprara, Phys. Rev. B 69, 184510 (2004).

17. M. Iskin and C.A.R. Sa de Melo, Phys. Rev. B 74, 144517 (2006).

18. L. Fanfarillo, L. Benfatto, S. Caprara, C. Castellani, and M. Grilli, Phys. Rev. B 79, 172508 (2009).

19. D. Innocenti, N. Poccia, A. Ricci, A. Valletta, S. Caprara, A. Perali, and A. Bianconi, Phys. Rev. B 82, 184528 (2010); A. Bianconi, D. Innocenti, A. Valletta, and A. Perali, J. Phys.: Conf. Ser. 529, 012007 (2014).

20. J. Ranninger, J.M. Robin, and M. Eschrig, Phys. Rev. Lett. 74, 4027 (1995).

21. A. Levchenko, M.R. Norman, and A.A. Varlamov, Phys. Rev. B 83, 020506(R) (2011).

22. L. Fanfarillo, L. Benfatto, and C. Castellani, Phys. Rev. B 85, 024507 (2012).

23. A. Guidini and A. Perali, Supercond. Sci. Technol. 27, 124002 (2014).

24. Q. Chen, J. Stajic, S. Tan, and K. Levin, Phys. Rep. 412, 188 (2005); Y. Shin, C.H. Schunck, A. Schirotzek, and W. Ketterle,
Nature 451, 689693 (2007); S. Giorgini, L.P. Pitaevskii, and S. Stringari, Rev. Mod. Phys. 80, 1215 (2008); F. Chevy and C. Mora, Rep. Prog. Phys. 73, 112401 (2010); Z. Shen, L. Radzihovsky, and V. Gurarie, Phys. Rev. Lett. 109, 245302 (2012); M. Randeria and E. Taylor, Ann. Rev. Condens. Matter Phys. 5, 209232 (2014).

25. J.P. Gaebler, Nat. Phys. 6, 569 (2010).

26. G.E. Volovik, The Universe in a Helium Droplet, Clarendon Press, Oxford (2003).

27. P. Ao and D.J. Thouless, Phys. Rev. Lett. 93, 090403-1 (2004); J.R. Aitchison, P. Ao, D.J. Thouless, and X.-M. Zhu, Phys. Rev. B 51, 6531 (1995).

28. J. Dziarmaga, Phys. Rev. B 53, 6572 (1996).

29. A. van Otterlo, M.V. Feigelman, V.B. Geshkenbein, and G. Blatter, Phys. Rev. Lett. 75, 3736 (1995); A. van Otterlo, D.S. Golubev, A.D. Zaikin, and G. Blatter, Eur. Phys. J. B 10, 131 (1999);

30. E. Simanek, Phys. Rev. B 52, 10336 (1995).

31. M. Stone, Phys. Rev. B 54, 13222 (1996).

32. N.B. Kopnin, G.E. Volovik, and U. Parts, Europhys. Lett. 32, 651 (1995).

33. J. Bardeen, R. Kummel, A.E. Jacobs, and L. Tewordt, Phys. Rev. 187, 556 (1969).

34. J. Bardeen and M.J. Stephen, Phys. Rev. 140, A 1197 (1965); P. Nozieres and W.F. Vinen, Philos. Mag. 14, 667 (1996).

35. N.B. Kopnin and V.E. Kravtsov, JETP Lett. 23, 578 (1976); N.B. Kopnin and M.M. Salomaa, Phys. Rev. B 44, 9667 (1991).

36. G.E. Volovik, JETP Lett. 65, 676 (1997).

37. F. Gaitan, Phys. Rev. B 51, 9061 (1995).

38. D. Mozyrsky, A.V. Chubukov, and M. Stone, to be submitted.

39. R.L. Stratonovich, Sov. Phys. Dokl. 2, 416 (1958); J. Hubbard, J. Phys. Rev. Lett. 3, 77 (1959); A. Altland and B. Simons, Condensed Matter Field Theory, University Press, Cambridge (2006).

40. L. He, H. Lu, G. Cao, H. Hu, and X.-J. Liu, Phys. Rev. A 92, 023620 (2015).

41. D.J. Scalapino, S.R. White, and S. Zhang, Phys. Rev. B 47, 7995 (1993); L. Benfatto, A. Toschi, S. Caprara, and C. Castellani, Phys. Rev. B 64, 140506(R) (2001); L. Benfatto, S. Caprara, C. Castellani, A. Paramekanti, and M. Randeria, Phys. Rev. B 63, 174513 (2001); S.G. Sharapov, V.P. Gusynin, and H. Beck, Eur. Phys. J. B 30, 45 (2002); L. Benfatto, A. Toschi, and S. Caprara, Phys. Rev. B 69, 184510 (2004).

42. Y. Lemonik, I.L. Aleiner, and B.L. Altshuler, arXiv:1505.04820. 